\title{
Strategy-proof location of public bads in a two-country model
}

Citation for published version (APA):

Lahiri, A., Peters, H. J. M., \& Storcken, A. J. A. (2015). Strategy-proof location of public bads in a twocountry model. Maastricht University, Graduate School of Business and Economics. GSBE Research Memoranda No. 007 https://doi.org/10.26481/umagsb.2015007

Document status and date:

Published: 01/01/2015

DOI:

10.26481/umagsb.2015007

Document Version:

Publisher's PDF, also known as Version of record

\section{Please check the document version of this publication:}

- A submitted manuscript is the version of the article upon submission and before peer-review. There can be important differences between the submitted version and the official published version of record.

People interested in the research are advised to contact the author for the final version of the publication, or visit the DOI to the publisher's website.

- The final author version and the galley proof are versions of the publication after peer review.

- The final published version features the final layout of the paper including the volume, issue and page numbers.

Link to publication

\footnotetext{
General rights rights.

- You may freely distribute the URL identifying the publication in the public portal. please follow below link for the End User Agreement:

www.umlib.nl/taverne-license

Take down policy

If you believe that this document breaches copyright please contact us at:

repository@maastrichtuniversity.nl

providing details and we will investigate your claim.
}

Copyright and moral rights for the publications made accessible in the public portal are retained by the authors and/or other copyright owners and it is a condition of accessing publications that users recognise and abide by the legal requirements associated with these

- Users may download and print one copy of any publication from the public portal for the purpose of private study or research.

- You may not further distribute the material or use it for any profit-making activity or commercial gain

If the publication is distributed under the terms of Article $25 \mathrm{fa}$ of the Dutch Copyright Act, indicated by the "Taverne" license above, 


\section{Maastricht University}

Abhinaba Lahiri, Hans Peters, Ton Storcken

Strategy-Proof Location of Public Bads in a Two-Country Model

$\mathrm{RM} / 15 / 007$

\section{GSBE}

Maastricht University School of Business and Economics

Graduate School of Business and Economics

P.O Box 616

NL-6200 MD Maastricht

The Netherlands 


\title{
Strategy-Proof Location of Public Bads in a Two-Country Model
}

\author{
Abhinaba Lahiri* $\quad$ Hans Peters ${ }^{\dagger} \quad$ Ton Storcken ${ }^{\ddagger}$
}

March 2015

\begin{abstract}
We consider the joint decision of placing public bads in each of two neighboring countries, modelled by two adjacent line segments. Residents of the two countries have single-dipped preferences, determined by the location of the nearest public bad to their dips. A social choice function or rule takes a profile of reported preferences as input and assigns the location of the public bad in each country. All rules satisfying strategy-proofness, country-specific Pareto optimality, non-corruptibility, and the far away condition are characterized. These rules pick only boundary locations.
\end{abstract}

Keywords: public bads, single-dipped preferences, strategy-proofness, noncorruptibility

\section{JEL Classification: D71}

\section{Introduction}

We consider two neighboring countries $A$ and $B$, represented by the real intervals $[-1,0]$ and $[0,1]$, respectively. Each country has to locate one public bad within its boundaries, and we assume that the countries jointly decide on these locations. As an example, think of the coast line shared by Netherlands and Germany: how can these countries jointly decide on the location of a windmill park in each country along this coast line? This location problem is one of public bads: public provisions that are beneficial for the countries but that no one likes to have in his backyard.

We assume that preferences are single-dipped in the sense that some one's preference is determined by the distance of this dip to the nearest location of a public bad. There are finitely many residents in each of the two countries. A

\footnotetext{
*Department of Quantitative Economics, Maastricht University, The Netherlands. Email: a.lahiri@maastrichtuniversity.nl

${ }^{\dagger}$ Department of Quantitative Economics, Maastricht University, The Netherlands. Email: h.peters@maastrichtuniversity.nl

‡Department of Quantitative Economics, Maastricht University, The Netherlands. Email: t.storcken@maastrichtuniversity.nl
} 
social choice function or rule will take the dips of all the residents of the two countries (called a profile) as input, and give a pair in $[-1,0] \times[0,1]$ as output. In this paper we characterize the class of all rules that simultaneously satisfy four conditions: strategy-proofness, non-corruptibility, country-specific Pareto optimality, and the far away condition.

The notion of strategy-proofness is the usual one, as introduced by Gibbard (1973) and Satterthwaite (1975): it can never be beneficial not to report one's true preference. Strategy-proofness ensures that decisions are based on the correct preference information. Although, in this context, a resident's home is a likely candidate for his dip, we still assume that residents may want to vote differently. For instance, they may regard the school of their children, or some important public area, as their dip.

Our version of Pareto optimality is stronger than the usual notion: we impose Pareto optimality specific to each country.

The non-corruptibility property is based on the similar notion introduced by Ritz (1985). This property ensures that after a unilateral deviation of a resident, if he remains indifferent between the old outcome and the new outcome both according to his true preference and according to his deviating preference, then both these outcomes must be the same. This means that a unilaterally deviating resident cannot change the outcome and remain unaffected by this change; i.e., a resident by unilateral deviation cannot make some other residents better off or worse off without affecting himself.

The far away condition implies, for instance, that if all the agents are indifferent, the rule selects $(-1,1)$ as the outcome. More generally, this condition states that if no resident strictly opposes the decision of placing one bad at the extreme end of the corresponding country, irrespective of the location of the other bad, then that bad should be placed at the extreme end. This condition can be thought of as a tie breaking condition ensuring that the outcome remains at one of the corner points of $[-1,0] \times[0,1]$.

We show that the range of rule satisfying these four properties consists of the corner points of $[-1,0] \times[0,1]$; thus, such a rule never places a public bad of a country in the interior of that country. As an example, consider the following rule (for simplicity, we assume that agents cannot have dips at -0.5 or at 0.5 ; and the total number of agents is odd). The rule selects $(-1,1)$ if there is a majority in favor of $(-1,1)$. If that is not the case and there are agents with dips in $[-1,-0.5)$, but there are no agents with dips in $(0.5,1]$, then the rule selects $(0,1)$. For the symmetrically opposite case where there are no agents with dips in $[-1,-0.5)$, but there are agents with dips in $(0.5,1]$, the rule selects $(-1,0)$. In all other cases, the rule selects $(0,0)$. This rule satisfies the four conditions. In general, the class of rules that satisfy the four properties is characterized on the basis of a family of pairs of coalitions; one coalition consisting of agents who strictly prefer $(-1,1)$ to all the other corner points of $[-1,0] \times[0,1]$, the other coalition consisting of agents who are indifferent among all the corner points of $[-1,0] \times[0,1]$. With such a family a rule is associated, and this rule satisfies the four conditions if and only if the family of coalition pairs satisfies certain conditions, including a monotonicity condition. 
This is a positive result as compared to the seminal impossibility theorem of Gibbard (1973) and Satterthwaite (1975), which says that if there are three or more alternatives, then it is impossible to find a non-dictatorial social choice function which is also strategy proof and Pareto optimal. One way out from this impossibility result is to consider restricted preference domains, where the restriction concerns both the true and the reported preferences. One possible restricted domain is the single-dipped preference domain. Peremans and Storcken (1999) have shown the equivalence between individual and group strategyproofness in subdomains of single-dipped preferences. Manjunath (2014) has characterized the class of all nondictatorial, strategy-proof and Pareto optimal social choice functions when preferences are single-dipped over an interval. Barberà, Berga and Moreno (2012) have characterized the class of all nondictatorial, group strategy-proof and Pareto optimal social choice functions when preferences are single-dipped over a line. The rules in the present paper bear similarities to the rules in the last two papers.

But there are impossibility results in this domain as well. Öztürk, Peters and Storcken $(2013,2014)$ have shown that there does not exist any nondictatorial social choice function that is strategy-proof and Pareto optimal when preferences are single-dipped over a disk, and over some, but not all, convex polytopes in the plane.

All these results are about strategy-proof location of one public bad. As far as we know, the present paper is the first one to consider the location of public bads in neigboring regions or countries. There is also a literature adopting a mechanism design approach to the location of public bads, that is, including monetary sidepayments: e.g., recently, Lescop (2007) and Sakai (2012), but we are not aware of results in this area addressing the location in more than one region.

This paper is organized as follows. Section 2 introduces the model and some preliminary results. Section 3 shows that internal locations are excluded, and Section 4 provides the characterization of all rules satisfying our conditions. Section 5 concludes with some examples, including examples showing logical independence of the four conditions.

\section{The two country model}

Let country $A$ be represented by the interval $[-1,0]$ and country $B$ by $[0,1]$. The set of possible alternatives is denoted by $\mathcal{A}=[-1,0] \times[0,1]$. The set of agents $N$ is partitioned in the set $N_{A}$ of inhabitants of country $A$ and the set $N_{B}$ of inhabitants of country $B$. Let the cardinalities of $N, N_{A}$ and $N_{B}$ be natural numbers $n, n_{A}, n_{B}$, with $n=n_{A}+n_{B}$.

Each agent $i \in N$ has a preference $R_{x(i)}$ over $\mathcal{A}$, characterized by its $\operatorname{dip} x(i)$ in $[-1,1]$, such that alternative $\left(a_{1}, b_{1}\right)$ is at least as good as alternative $\left(a_{2}, b_{2}\right)$ at $R_{x(i)}$, with the usual notation $\left(a_{1}, b_{1}\right) R_{x(i)}\left(a_{2}, b_{2}\right)$, if

$$
\min \left\{\left|a_{1}-x(i)\right|,\left|b_{1}-x(i)\right|\right\} \geq \min \left\{\left|a_{2}-x(i)\right|,\left|b_{2}-x(i)\right|\right\} .
$$


As usual, $P_{x(i)}$ denotes the strict or asymmetric part of $R_{x(i)}$ and $I_{x(i)}$ denotes the indifference or symmetric part. Preference $R_{x(i)}$ is completely determined by its $\operatorname{dip} x(i)$. Therefore, preferences are identified with their dips and denoted by $x(i)$ instead of $R_{x(i)}{ }^{1}$

A preference profile $z$ assigns to each agent $i$ in $N$ a preference $z(i)$ such that $z(i)$ is a dip in $[-1,0]$ if $i \in N_{A}$ and in $[0,1]$ if $i \in N_{B}$. The set of all preference profiles is denoted by $\mathcal{R}$.

For a profile $z$ and a non-empty set $S \subseteq N$, let $z_{S}=(z(i))_{i \in S}$. For $i \in N$, profile $z^{\prime}$ is an $i$-deviation of $z$ if $z_{N \backslash\{i\}}=z_{N \backslash\{i\}}^{\prime}$. For $a \in A$ and $S \subseteq N_{A}$, $\left(a^{S}, z_{N \backslash S}\right)$ denotes the profile where all $i \in N \backslash S$ have preference $z(i)$ and all $i \in S$ have preference $a$. Similar notations will have similar meaning.

A rule $\varphi$ assigns to each preference profile $z$ an alternative $\varphi(z)=(\alpha(z), \beta(z))$ $\in \mathcal{A}$. For $x, y \in \mathbb{R}, \mu(x, y)=\frac{x+y}{2}$ denotes the midpoint of $x$ and $y$. In case there is no confusion, for a profile $z$ we write $\mu(z)$ instead of $\mu(\alpha(z), \beta(z))$.

We consider the following properties for a rule $\varphi$.

Strategy-Proofness (SP) $\varphi$ is strategy-proof if $\varphi(z) R_{z(i)} \varphi\left(z^{\prime}\right)$ for every $z \in \mathcal{R}$, every $i \in N$, and every $i$-deviation $z^{\prime}$ of $z$.

Strategy-proofness says that truth-telling is a weakly dominant strategy.

Non-Corruptibility (NC) $\varphi$ is non-corruptible if $\varphi(z)=\varphi\left(z^{\prime}\right)$ for every $z \in \mathcal{R}$, $i \in N$, and $i$-deviation $z^{\prime}$ of $z$ such that $\varphi(z) I_{z(i)} \varphi\left(z^{\prime}\right)$ and $\varphi(z) I_{z^{\prime}(i)} \varphi\left(z^{\prime}\right)$.

At non-corruptible rules a unilateral deviation either affects the deviator's preference somewhere or has no effect at all. This condition, introduced by Ritz (1984), eliminates tie-breaking caused by individual indifferences.

Country-Specific Pareto optimality (CPO) Rule $\varphi$ is Pareto optimal for country $A$ if for every profile $z$ there does not exist an $a \in[-1,0]$ such that $(a, \beta(z)) R_{z(i)} \varphi(z)$ for all $i \in N_{A}$ and $(a, \beta(z)) P_{z(k)} \varphi(z)$ for at least one $k \in N_{A}$. It is Pareto optimal for country $B$ if for every profile $z$ there does not exist a $b \in[0,1]$ such that $(\alpha(z), b) R_{z(i)} \varphi(z)$ for all $i \in N_{B}$ and $(\alpha(z), b) P_{z(i)} \varphi(z)$ for at least one $k \in N_{B}$. Rule $\varphi$ is country-specific Pareto optimal if it is both Pareto optimal for country $A$ and Pareto optimal for country $B$.

Far Away Condition (FA) Rule $\varphi$ satisfies the far away condition if for every profile $z$ :

- if $(\alpha(z), 1) R_{z(i)} \varphi(z)$ for all $i \in N$, then $\beta(z)=1$, and

- if $(-1, \beta(z)) R_{z(i)} \varphi(z)$ for all $i \in N$, then $\alpha(z)=-1$.

Monotonicity (MON) Rule $\varphi$ is monotone if $\varphi(z)=\varphi\left(z^{\prime}\right)$ for all $z, z^{\prime} \in \mathcal{R}$ such that for all agents $i \in N$ :

$$
\text { - } z^{\prime}(i) \leq z(i) \leq \alpha(z) \text { or }
$$

\footnotetext{
${ }^{1}$ In fact, $R_{x(i)}$ is a 'single trenched' preference. For example, suppose the dip of an agent in country $A$ is at -0.5 . Then $\{-0.5\} \times B$ is the 'trench' in his preference.
} 
- $\alpha(z) \leq z(i) \leq z^{\prime}(i) \leq \mu(z)$ or

- $\mu(z) \leq z^{\prime}(i) \leq z(i) \leq \beta(z)$ or

- $\beta(z) \leq z(i) \leq z^{\prime}(i)$.

Thus, $\varphi$ is monotone if outcomes do not change whenever agents increase their minimal distance to these locations while not jumping across any of these locations.

Remark 2.1 For an agent $i$ with $\operatorname{dip} z(i)$ the lower contour set at location $(a, b) \in \mathcal{A}$ is defined by $L((a, b), z(i))=\left\{(x, y) \in \mathcal{A}:(a, b) R_{z(i)}(x, y)\right\}$. Rule $\varphi$ is said to be Maskin monotone if $\varphi(z)=\varphi\left(z^{\prime}\right)$ for all profiles $z$ and $z^{\prime}$ such that $L(\varphi(z), z(i)) \subseteq L\left(\varphi(z), z^{\prime}(i)\right)$ for all $i \in N$. Evidently, Maskin monotonicity implies monotonicity as defined above. To see that the reverse does not hold consider a profile $z$ such that there exists at least one agent $i \in N_{A}$ with $z(i)=$ -1 , and suppose that $-1<\alpha(z) \leq 0$. Consider the $i$-deviation $z^{\prime}$ of $z$ such that $z^{\prime}(i)=\mu(-1, \alpha(z))$. Note that $L(\varphi(z), z(j))=L\left(\varphi(z), z^{\prime}(j)\right)$ for all agents $j$, so that Maskin monotonicity would imply $\varphi\left(z^{\prime}\right)=\varphi(z)$. Monotonicity in our sense, however, allows $\alpha\left(z^{\prime}\right)$ to be equal to -1 .

The following lemma shows that monotonicity is implied by strategy-proofness and non-corruptibility.

Lemma 2.2 Let $\varphi$ satisfy SP and NC. Then $\varphi$ satisfies MON.

Proof. It is sufficient to prove monotonicity for an $i$-deviation from $z \in \mathcal{R}$ to $z^{\prime} \in \mathcal{R}$ for an agent $i \in N_{A}$. There are three cases.

(a) $z^{\prime}(i)<z(i) \leq \alpha(z)$.

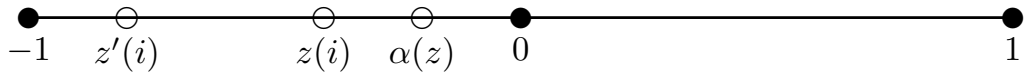

If agent $i$ deviates from $z(i)$ to $z^{\prime}(i)$ then SP implies

$$
|z(i)-\alpha(z)| \geq\left|z(i)-\alpha\left(z^{\prime}\right)\right|
$$

and if agent $i$ deviates from $z^{\prime}(i)$ to $z(i)$ then SP implies

$$
\left|z^{\prime}(i)-\alpha\left(z^{\prime}\right)\right| \geq\left|z^{\prime}(i)-\alpha(z)\right| .
$$

If $x \in \mathbb{R}$ is such that $z(i)=\mu(x, \alpha(z))$ then by $(1): \alpha\left(z^{\prime}\right) \in[\max \{x,-1\}, \alpha(z)]$, hence by $(2)$ : $\alpha\left(z^{\prime}\right)=\alpha(z)$. By NC, $\varphi(z)=\varphi\left(z^{\prime}\right)$.

(b) $\alpha(z) \leq z(i)<z^{\prime}(i) \leq \mu(z)$.

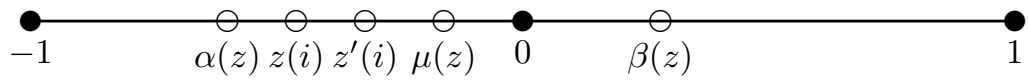


If agent $i$ deviates from $z(i)$ to $z^{\prime}(i)$ then SP implies

$$
|z(i)-\alpha(z)| \geq\left|z(i)-\alpha\left(z^{\prime}\right)\right| \text { or }|z(i)-\alpha(z)| \geq\left|z(i)-\beta\left(z^{\prime}\right)\right|
$$

and if agent $i$ deviates from $z^{\prime}(i)$ to $z(i)$ then SP implies

$$
\left|z^{\prime}(i)-\alpha\left(z^{\prime}\right)\right| \geq\left|z^{\prime}(i)-\alpha(z)\right| \text { and }\left|z^{\prime}(i)-\beta\left(z^{\prime}\right)\right| \geq\left|z^{\prime}(i)-\alpha(z)\right| \text {. }
$$

If the first inequality in (3) holds then the first inequality in (4) implies $\alpha\left(z^{\prime}\right)=$ $\alpha(z)$. By the second inequality in (4), $\alpha(z)=\alpha\left(z^{\prime}\right)$ is closer to both $z(i)$ and $z^{\prime}(i)$ than $\beta^{\prime}(z)$ is, so by NC, $\varphi(z)=\varphi\left(z^{\prime}\right)$. If the second inequality in (3) holds then the second inequality in (4) is violated, a contradiction.

(c) $\alpha(z) \leq \mu(z) \leq z^{\prime}(i)<z(i)$.

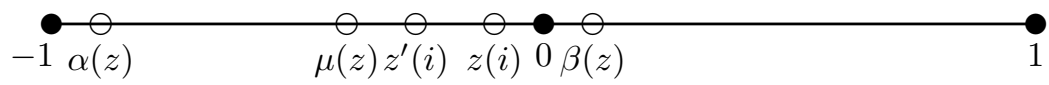

If agent $i$ deviates from $z(i)$ to $z^{\prime}(i)$ then SP implies

$$
|z(i)-\beta(z)| \geq\left|z(i)-\alpha\left(z^{\prime}\right)\right| \text { or }|z(i)-\beta(z)| \geq\left|z(i)-\beta\left(z^{\prime}\right)\right|
$$

and if agent $i$ deviates from $z^{\prime}(i)$ to $z(i)$ then SP implies

$$
\left|z^{\prime}(i)-\alpha\left(z^{\prime}\right)\right| \geq\left|z^{\prime}(i)-\beta(z)\right| \text { and }\left|z^{\prime}(i)-\beta\left(z^{\prime}\right)\right| \geq\left|z^{\prime}(i)-\beta(z)\right| \text {. }
$$

If the first inequality in (5) holds then the first inequality in (6) implies $\alpha\left(z^{\prime}\right)=$ $\beta(z)$, hence $\alpha\left(z^{\prime}\right)=\beta(z)=0$. Hence by NC, $\varphi(z)=\varphi\left(z^{\prime}\right)$. If the second inequality in (5) holds then the second inequality in (6) implies $\beta(z)=\beta\left(z^{\prime}\right)$. Then both at $z(i)$ and $z^{\prime}(i)$ agent $i$ is indifferent between $\varphi(z)$ and $\varphi\left(z^{\prime}\right)$, so that by $\mathrm{NC}, \varphi(z)=\varphi\left(z^{\prime}\right)$.

\section{$3 \quad$ No internal solutions}

In this section show that under the conditions imposed in this paper a rule never assigns locations in the interiors of the two countries.

Theorem 3.1 Let rule $\varphi$ satisfy SP, CPO, $N C$, and FA. Then $\varphi(z) \in\{(-1,1)$, $(0,0),(-1,0),(0,1)\}$ for every $z \in \mathcal{R}$.

The proof of this theorem uses the two lemmas below. For the rest of this section we assume that $\varphi$ is a rule satisfying the four conditions in Theorem 3.1. For a profile $z \in \mathcal{R}$ we define $S(z)=\left\{i \in N_{A}: z(i) \geq \alpha(z)\right\}$ and $T(z)=\{i \in$ $\left.N_{B}: z(i) \leq \beta(z)\right\}$. By Lemma 2.2, $\varphi$ is monotone. Therefore we may assume that
(a) $z=\left(-1^{N_{A} \backslash S(z)}, 0^{S(z)}, \mu(z)^{T(z)}, 1^{N_{B} \backslash T(z)}\right)$ if $\mu(z) \in[0,1]$, and
(b) $z=\left(-1^{N_{A} \backslash S(z)}, \mu(z)^{S(z)}, 0^{T(z)}, 1^{N_{B} \backslash T(z)}\right)$ if $\mu(z) \in[-1,0]$. 
The following lemma shows that if one of the two bads is located at 0 , then the other one cannot be located at an interior point of its country.

\section{Lemma 3.2}

(a) Let $\alpha(z)=0<\beta(z)$. Then $z(i) \leq \frac{1}{2}$ for all $i \in N$, and $\beta(z)=1$.

(b) Let $\alpha(z)<0=\beta(z)$. Then $z(i) \geq-\frac{1}{2}$ for all $i \in N$, and $\alpha(z)=-1$.

Proof. We only prove part (a), part (b) is analogous. Let $\alpha(z)=0<\beta(z)$. Then $\mu(z) \in[0,1]$, so $z=\left(-1^{N_{A} \backslash S(z)}, 0^{S(z)}, \mu(z)^{T(z)}, 1^{N_{B} \backslash T(z)}\right)$. Since all agents $i \in T(z)$ are indifferent between $(0,0)$ and $(0, \beta(z))$ and all agents $i \in N_{B} \backslash T(z)$ strictly prefer $(0,0)$ to $(0, \beta(z))$, CPO implies that $T(z)=N_{B}$. From this, $\beta(z)=1$ follows by FA, and thus $z(i) \leq \frac{1}{2}$ for all $i \in N$.

The next lemma shows that if one of the two bads is located at an extreme end, then the other is located at an extreme end as well.

Lemma $3.3 \alpha(z) \in\{-1,0\}$ if and only if $\beta(z) \in\{0,1\}$.

Proof. We show the if-direction, the other direction is analogous. By Lemma 3.2 (b) it is sufficient to prove that $\alpha(z) \in\{-1,0\}$ if $\beta(z)=1$. To the contrary suppose $-1<\alpha(z)<0$ and $\beta(z)=1$. Then $0<\mu(z)<\frac{1}{2}$ and $T(z)=N_{B}$ by definition of $T(z)$, so that $z=\left(-1^{N_{A} \backslash S(z)}, 0^{S(z)}, \mu(z)^{N_{B}}\right)$. By FA, $\beta\left(z_{N_{A}}^{\prime}, \mu(z)^{N_{B}}\right)=1$ for all profiles $z^{\prime}$. We will compare the four profiles in the following table:

$$
\begin{array}{cccccc} 
& N_{A} \backslash S(z) & S(z) & N_{B} & \alpha & \beta \\
z & -1 & 0 & \mu(z) & \alpha(z) & 1 \\
z^{-} & \frac{\alpha(z)-1}{2} & 0 & \mu(z) & \alpha\left(z^{-}\right) & 1 \\
z^{*} & \frac{\alpha(z)-1}{2} & \frac{\alpha(z)}{2} & \mu(z) & \alpha\left(z^{*}\right) & 1 \\
z^{+} & -1 & \frac{\alpha(z)}{2} & \mu(z) & \alpha\left(z^{+}\right) & 1
\end{array}
$$

(For instance, the first line of this table means that $z=\left(-1^{N_{A} \backslash S(z)}, 0^{S(z)}\right.$, $\mu(z)^{N_{B}}$ ) and that $\beta(z)=1$. Note that to all these profiles $\beta$ assigns location 1 by FA.) Consider profiles $z$ and $z^{-}$. SP implies that $\alpha\left(z^{-}\right) \in\{\alpha(z),-1\}$, and then CPO implies that $\alpha\left(z^{-}\right)=-1$. Applying SP at profiles $z^{-}$and $z^{*}$ now yields that $\alpha\left(z^{*}\right)=-1$. Considering SP at the profiles $z$ and $z^{+}$yields that $\alpha\left(z^{+}\right) \in\{\alpha(z), 0\}$, and then CPO implies $\alpha\left(z^{+}\right)=0$. Finally, comparing profiles $z^{+}$and $z^{*}$ yields a contradiction with SP since $(0,1)=\varphi\left(z^{+}\right)$is better for $\operatorname{dip} \frac{\alpha(z)-1}{2}$ than $(-1,1)=\varphi\left(z^{*}\right)$.

Proof of Theorem 3.1. Suppose that $-1<\alpha(z)<0$ and $0<\beta(z)<$ 1. We will derive a contradiction: then the proof is complete by Lemma 3.3. We assume without loss of generality that $\mu(z) \in[0,1]$, so that $z=$ $\left(-1^{N_{A} \backslash S(z)}, 0^{S(z)}, \mu(z)^{T(z)}, 1^{N_{B} \backslash T(z)}\right)$. 
First note that $S(z) \neq \emptyset, S(z) \neq N_{A}, T(z) \neq \emptyset$, and $T(z) \neq N_{B}$ by CPO. For $t=1,2, \ldots$ we define profiles in the following table:

$\begin{array}{ccccc} & N_{A} \backslash S(z) & S(z) & T(z) & N_{B} \backslash T(z) \\ z^{0}=z & -1 & 0 & \mu(z) & 1 \\ z^{t} & -1 & \frac{\alpha\left(z^{t-1}\right)}{2} & \mu(z) & 1 \\ v^{1} & \frac{\alpha\left(z^{0}\right)-1}{2} & 0 & \mu(z) & 1 \\ v^{t} & \frac{\alpha\left(z^{t-1}\right)-1}{2} & 0 & \mu(z) & 1 \\ w^{t} & \frac{\alpha\left(z^{t-1}\right)-1}{2} & \frac{\alpha\left(z^{t-1}\right)}{2} & \mu(z) & 1\end{array}$

The proof now proceeds in a few steps.

Step 1. Let $t \geq 1$ and suppose that $-1<\alpha\left(z^{t-1}\right)<0$. Then $\varphi\left(v^{t}\right)=\varphi\left(w^{t}\right)=$ $(-1,1)$.

Proof. Comparing $z^{t-1}$ and $v^{t}$, SP implies $\alpha\left(v^{t}\right) \leq \alpha\left(z^{t-1}\right)$ since otherwise $N_{A} \backslash S(z)$ can manipulate from $z^{t-1}$ to $v^{t}$, and therefore $\alpha\left(v^{t}\right) \in\left\{-1, \alpha\left(z^{t-1}\right)\right\}$ since otherwise $N_{A} \backslash S(z)$ can manipulate from $v^{t}$ to $z^{t-1}$. By CPO, this implies $\alpha\left(v^{t}\right)=-1$. Therefore, by Lemma 3.3, $\beta\left(v^{t}\right) \in\{0,1\}$. Now Lemma 3.2(b) implies that $\beta\left(v^{t}\right) \neq 0$. Thus, $\beta\left(v^{t}\right)=1$ and $\varphi\left(v^{t}\right)=(-1,1)$. By SP, going from $w^{t}$ to $v^{t}$, we obtain $\alpha\left(w^{t}\right)=-1$. By Lemma 3.3, $\beta\left(w^{t}\right) \in\{0,1\}$. Since $\frac{\alpha\left(z^{t-1}\right)}{2}>-\frac{1}{2}$, Lemma 3.2(b) then implies $\beta\left(w^{t}\right)=1$. Thus, $\varphi\left(w^{t}\right)=(-1,1)$.

Step 2. For all $t \geq 1$ :

$$
-1<\alpha\left(z^{t}\right)<\alpha\left(z^{t-1}\right)<\ldots<0 \leq 2 \mu(z)<\beta\left(z^{t}\right) \leq-\alpha\left(z^{t-1}\right)<1 .
$$

Proof. By assumption we have $-1<\alpha\left(z^{0}\right)=\alpha(z)<0 \leq 2 \mu(z)<\beta\left(z^{0}\right)=$ $\beta(z)<1$. We prove the statement in Step 2 by induction. Assume it is true for all $s<t$, where $t \geq 1$. By going from $z^{t}$ to $z^{t-1}$, SP implies $\alpha\left(z^{t}\right) \in\left[-1, \alpha\left(z^{t-1}\right)\right]$ or $\alpha\left(z^{t}\right)=0$. Suppose that $\alpha\left(z^{t}\right)=0$, then by Lemma 3.2(a) we must have $\beta\left(z^{t}\right)=0$; by SP, going from $w^{t}$ to $z^{t}$, this contradicts $\varphi\left(w^{t}\right)=(-1,1)$ as established in Step 1. Hence, $\alpha\left(z^{t}\right) \in\left[-1, \alpha\left(z^{t-1}\right)\right]$. Now CPO applied to the profile $z^{t}$ implies $\alpha\left(z^{t}\right) \neq \alpha\left(z^{t-1}\right)$, so $\alpha\left(z^{t}\right) \in\left[-1, \alpha\left(z^{t-1}\right)\right)$. In turn, this and the induction hypothesis imply by SP, going from $z^{t-1}$ to $z^{t}$, that $\beta\left(z^{t}\right) \leq-\alpha\left(z^{t-1}\right)$. Then $\alpha\left(z^{t}\right)>-1$, since otherwise by Lemma 3.3, $\beta\left(z^{t}\right)=0$, contradicting Lemma 3.2(b), or $\beta\left(z^{t}\right)=1$, contradicting that $-\alpha\left(z^{t-1}\right)<1$ by the induction hypothesis. Finally, $2 \mu(z)<\beta\left(z^{t}\right)$ follows by CPO.

Step 3. If $\mu(z)=0$ then $\mu\left(z^{1}\right) \neq 0$.

Proof. Follows from Step 2 by taking $t=1$.

Step 4. $\mu(z)=0$.

Proof. First suppose that for some $t>1, \mu\left(z^{t}\right) \geq \frac{\alpha\left(z^{t-2}\right)}{2}$. Then, by Step 2, for all $i \in S(z)$ we have $\alpha\left(z^{t}\right)<\frac{\alpha\left(z^{t-1}\right)}{2}=z^{t}(i)<z^{t-1}(i)=\frac{\alpha\left(z^{t-2}\right)}{2} \leq \mu\left(z^{t}\right)$, so that by MON we have $\varphi\left(z^{t-1}\right)=\varphi\left(z^{t}\right)$, a contradiction. Thus, $\mu\left(z^{t}\right)<\frac{\alpha\left(z^{t-2}\right)}{2}$ for all $t>1$. Hence, $\alpha\left(z^{t}\right)+\beta\left(z^{t}\right)<\alpha\left(z^{t-2}\right)$ for all $t>1$. By Step 2 this 
implies $2 \mu(z)<\beta\left(z^{t}\right)<\alpha\left(z^{t-2}\right)-\alpha\left(z^{t}\right)$, which implies that $\mu(z)=0$ since $\alpha\left(z^{t-2}\right)-\alpha\left(z^{t}\right)$ converges to 0 for $t$ going to infinity.

We can now complete the proof of the theorem. Step 4 implies that $\mu(z)=0$ for any profile $z$ with $\alpha(z) \in(-1,0)$ and $\beta(z) \in(0,1)$. This contradicts Step 3 since $z^{1}$ is also such a profile.

\section{The rules}

In this section, we describe the class of rules $\varphi$ that satisfy CPO, SP, NC, and FA. Theorem 3.1 says that the range of such rules $\varphi$ is equal to $\mathcal{B}=$ $\{-11,-10,01,00\}$, where -11 denotes $(-1,1)$, etc. For a profile $z \in \mathcal{R}$, the restriction of $z$ to $\mathcal{B}$ is denoted by $\left.z\right|_{\mathcal{B}}$. We first show that for such rules $\varphi$ only these restricted preferences matter.

Lemma 4.1 Let rule $\varphi$ satisfy $C P O, S P, N C$, and $F A$, and let $z, z^{\prime} \in \mathcal{R}$ such that $\left.z\right|_{\mathcal{B}}=\left.z^{\prime}\right|_{\mathcal{B}}$. Then $\varphi(z)=\varphi\left(z^{\prime}\right)$.

Proof. Without loss of generality we may assume that $z$ and $z^{\prime}$ are $i$-deviations. Theorem 3.1 implies that $\varphi(z)$ and $\varphi\left(z^{\prime}\right)$ are both in $\mathcal{B}$. Since $\left.z\right|_{\mathcal{B}}=\left.z^{\prime}\right|_{\mathcal{B}}$, agent $i$ in $N$ has at $z(i)$ the same preference between $\varphi(z)$ and $\varphi\left(z^{\prime}\right)$ as at $z^{\prime}(i)$. By $\mathrm{SP}$, agent $i$ must be indifferent between $\varphi(z)$ and $\varphi\left(z^{\prime}\right)$ both at $z(i)$ and $z^{\prime}(i)$. Hence $\varphi(z)=\varphi\left(z^{\prime}\right)$ by NC.

On $\mathcal{B}$ there are just four different single-dipped preferences. These preferences, with dip $x$ and symmetric and asymmetric parts $\sim$ and $\succ$, are the following:

- If $-1 \leq x<0.5$, then $00 \sim 01 \succ-11 \sim-10$.

- If $x \in\{-0.5,0.5\}$, then $00 \sim 01 \sim-11 \sim-10$.

- If $-0.5<x<0.5$, then $-11 \succ-10 \sim 00 \sim 01$.

- If $0.5<x \leq 1$, then $-10 \sim 00 \succ-11 \sim 01$.

We will show that each rule satisfying CPO, SP, NC, and FA, is determined by monotonic voting between -11 and 00 , except for cases where -11 and 00 cannot be selected because of FA or CPO. These voting rules are characterized by families of decisive pairs of coalitions of agents. The first coalition of such a pair contains the agents with dip is strictly between -0.5 and 0.5 : these agents strictly prefer outcome -11 over outcome 00 . The second coalition contains the agents with dip either -0.5 or 0.5 : these agents are indifferent between -11 and 00. We will now make this more precise.

Definition $4.2 \mathcal{W} \subseteq 2^{N} \times 2^{N}$ is a family of decisive pairs if

(D) $U$ and $V$ are disjoint for all $(U, V) \in \mathcal{W}$; 
(M) Monotonicity: $\left(U^{\prime}, V^{\prime}\right) \in \mathcal{W}$ whenever $\left(U^{\prime}, V^{\prime}\right) \in 2^{N} \times 2^{N}$ and there exist $(U, V) \in \mathcal{W}$ such that $U \subseteq U^{\prime}$ and $U \cup V \subseteq U^{\prime} \cup V^{\prime}$;

(B) Boundary conditions:

(a) $(U, V) \in \mathcal{W}$ whenever $U \cup V=N$ and $U \cap V=\emptyset$,

(b) $(U, V) \notin \mathcal{W}$ if $U \cap N_{A}=\emptyset$ and $N_{A} \nsubseteq V$ or if $U \cap N_{B}=\emptyset$ and $N_{B} \nsubseteq V$.

In order to understand where this definition comes from, for a profile $z \in \mathcal{R}$ let $U(z)=\{i \in N:-0.5<z(i)<0.5\}$ be the set of agents who strictly prefer -11 to 00 ; and let $V(z)=\{i \in N: z(i) \in\{-0.5,0.5\}\}$ be the set of agents who are indifferent between -11 and 00 . For a rule $\varphi$ define

$$
\mathcal{W}_{\varphi}=\{(U(z), V(z)): z \in \mathcal{R}, \varphi(z)=-11\}
$$

We now have:

Lemma 4.3 Let $\varphi$ satisfy SP, CPO, NC, and FA. Then $\mathcal{W}_{\varphi}$ is a family of decisive pairs.

Proof. Condition (D) follows from the definition of $U(z)$ and $V(z)$. For condition (M), let $z \in \mathcal{R}$ with $\varphi(z)=-11$. Consider a $j$-deviation $z^{\prime}$ of $z$ such that $U(z) \subseteq U\left(z^{\prime}\right)$ and $U(z) \cup V(z) \subseteq U\left(z^{\prime}\right) \cup V\left(z^{\prime}\right)$. It is sufficient to prove that $\varphi\left(z^{\prime}\right)=-11$. Without loss of generality assume that $j \in N_{A}$. If $U(z)=U\left(z^{\prime}\right)$ and $V(z)=V\left(z^{\prime}\right)$, then we are done by Lemma 4.1. Assume $U(z) \neq U\left(z^{\prime}\right)$ or $V(z) \neq V\left(z^{\prime}\right)$. Since $U(z) \subseteq U^{\prime}\left(z^{\prime}\right)$ and $U(z) \cup V(z) \subseteq U\left(z^{\prime}\right) \cup V\left(z^{\prime}\right)$ it follows that $z(j) \leq z^{\prime}(j) \leq 0$. So by MON, $\varphi$ implies $\varphi\left(z^{\prime}\right)=\varphi(z)=-11$.

For condition (B), part (a), let $U, V \subseteq N$ such that $U \cap V=\emptyset$ and $U \cup V=N$. Consider the profile $z \in \mathcal{R}$ with $z(i)=0$ for all $i \in U, z(i)=-0.5$ for all $i \in V \cap N_{A}$, and $z(i)=0.5$ for all $i \in V \cap N_{B}$. Then $\varphi(z)=-11$ by CPO and/or FA. Hence $(U, V)=(U(z), V(z)) \in \mathcal{W}_{\varphi}$. For part (b), let $(U, V) \in 2^{N} \times 2^{N}$ and suppose that $U \cap N_{A}=\emptyset$ and $N_{A} \nsubseteq V$ (the other case is similar). Let $z$ be any profile with $(U(z), V(z))=(U, V)$. Then $z(i) \leq-0.5$ for all $i \in N_{A}$ and $z(i)<-0.5$ for some $i \in N_{A}$. By CPO, $\alpha(z)=0$. Hence, $(U, V) \notin \mathcal{W}_{\varphi}$.

Conversely, for a family of decisive pairs $\mathcal{W}$ a rule $\varphi_{\mathcal{W}}$ is defined as follows. Let $z \in \mathcal{R}$, then:

$$
\varphi_{\mathcal{W}}(z)=\left\{\begin{array}{cl}
-11 & \text { if }(U(z), V(z)) \in \mathcal{W} \\
-10 & \text { if }(U(z), V(z)) \notin \mathcal{W} \text { and } N_{A} \subseteq U(z) \cup V(z) \\
01 & \text { if }(U(z), V(z)) \notin \mathcal{W} \text { and } N_{B} \subseteq U(z) \cup V(z) \\
00 & \text { otherwise. }
\end{array}\right.
$$

In words, $\varphi_{\mathcal{W}}$ assigns -11 to a profile $z$ if the pair $(U(z), V(z))$ is decisive. Otherwise, it assigns 00 unless FA demands otherwise, that is, -10 or 01 . Next, we prove that $\varphi_{\mathcal{W}}$ satisfies our four conditions. 
Lemma 4.4 Let $\mathcal{W}$ be a family of decisive pairs. Then $\varphi_{\mathcal{W}}$ satisfies SP, CPO, $N C$, and $F A$.

Proof. We first prove SP of $\varphi_{\mathcal{W}}$. Consider $z \in \mathcal{R}$ and an $i$-deviation $z^{\prime}$ of $z$ for $i \in N_{A}$. It is sufficient to prove that $i$ weakly prefers $\varphi_{\mathcal{W}}(z)$ to $\varphi_{\mathcal{W}}\left(z^{\prime}\right)$. This is evidently the case if $\varphi_{\mathcal{W}}(z)=\varphi_{\mathcal{W}}\left(z^{\prime}\right)$ or if $z(i)=-0.5$. Therefore assume that $\varphi_{\mathcal{W}}(z) \neq \varphi_{\mathcal{W}}\left(z^{\prime}\right)$ and that $z(i) \neq-0.5$. We distinguish the following two cases.

- $-1 \leq z(i)<-0.5$. Then $U(z) \subseteq U\left(z^{\prime}\right)$ and $U(z) \cup V(z) \subseteq U\left(z^{\prime}\right) \cup V\left(z^{\prime}\right)$ and because of $\varphi_{\mathcal{W}}(z) \neq \varphi_{\mathcal{W}}\left(z^{\prime}\right)$ at least one of these inclusions is strict. Hence, $(\mathrm{M})$ of $\mathcal{W}$ and $\varphi_{\mathcal{W}}(z) \neq \varphi_{\mathcal{W}}\left(z^{\prime}\right)$ imply $\varphi_{\mathcal{W}}(z) \neq-11$. Since $N_{A} \nsubseteq$ $U(z) \cup V(z)$, we have $\varphi_{\mathcal{W}}(z) \neq-10$. Hence, $\varphi_{\mathcal{W}}(z) \in\{00,01\}$, so that $i$ at $z(i)$ weakly prefers $\varphi_{\mathcal{W}}(z)$ to $\varphi_{\mathcal{W}}\left(z^{\prime}\right)$.

- $-0.5<z(i) \leq 0$. Then $U\left(z^{\prime}\right) \subseteq U(z)$ and $U\left(z^{\prime}\right) \cup V\left(z^{\prime}\right) \subseteq U(z) \cup V(z)$, and because of $\varphi_{\mathcal{W}}(z) \neq \varphi_{\mathcal{W}}\left(z^{\prime}\right)$ at least one of these inclusions is strict. If $\varphi_{\mathcal{W}}(z) \in\{-10,00,01\}$ then by $(\mathrm{M})$ of $\mathcal{W}$ and the definition of $\varphi_{\mathcal{W}}$ we have $\varphi_{\mathcal{W}}(z)=\varphi_{\mathcal{W}}\left(z^{\prime}\right)$, a contradiction. Hence $\varphi_{\mathcal{W}}(z)=-11$, which is the single best outcome at $z(i)$.

We next prove CPO of $\varphi_{\mathcal{W}}$. It is sufficient to prove this for country $A$. To the contrary, suppose that all agents in $N_{A}$ weakly prefer $\left(a, \beta_{\mathcal{W}}(z)\right)$ to $\varphi_{\mathcal{W}}(z)=$ $\left(\alpha_{\mathcal{W}}(z), \beta_{\mathcal{W}}(z)\right)$ and some $j$ in $N_{A}$ strictly. We distinguish three cases.

- $\alpha_{\mathcal{W}}(z)=0$. Then $(U(z), V(z)) \notin \mathcal{W}$ and all agents in $N_{A}$ have their dip equal to or greater than $-\frac{a}{2} \geq-\frac{1}{2}$. Hence $N_{A} \subseteq U(z) \cup V(z)$, which implies $\alpha_{\mathcal{W}}(z)=-1$, a contradiction.

- $\beta_{\mathcal{W}}(z)=0$ and $\alpha_{\mathcal{W}}(z)=-1$. Then $(U(z), V(z)) \notin \mathcal{W}$ and $N_{A} \subseteq U(z) \cup$ $V(z)$. But then all agents in $N_{A}$ have their dip greater than or equal to $-\frac{1}{2}$, which contradicts the existence of agents $j$ who strictly prefer $(a, 0)$ to -10 .

- $\beta_{\mathcal{W}}(z)=1$ and $\alpha_{\mathcal{W}}(z)=-1$. Then $(U(z), V(z)) \in \mathcal{W}$. So, by condition (B)(b) of $\mathcal{W}$ there are agents $i \in N_{A} \cap U(z)$ or all agents in $N_{A}$ have their dip at $-\frac{1}{2}$. Since agents in $N_{A} \cap U(z)$ strictly prefer -11 to every other outcome $(a, 1)$, we must have that that all agents in $N_{A}$ have their dip at $-\frac{1}{2}$. At dip $-\frac{1}{2}$, however, outcome -11 is weakly preferred to every outcome $(a, 1)$ for $-1 \leq a \leq 0$. This contradicts the existence of agents $j$ who strictly prefer $(a, 1)$ to -11 .

Third, we prove $\mathrm{NC}$ of $\varphi_{\mathcal{W}}$. Consider $z \in \mathcal{R}$ and an $i$-deviation $z^{\prime}$ of $z$ for $i \in N_{A}$, and suppose that $i$ is indifferent between $\varphi_{\mathcal{W}}(z)$ and $\varphi_{\mathcal{W}}\left(z^{\prime}\right)$ both at $z(i)$ and at $z^{\prime}(i)$. It is sufficient to prove that $\varphi_{\mathcal{W}}(z)=\varphi_{\mathcal{W}}\left(z^{\prime}\right)$. We may assume that $z(i)<z^{\prime}(i)$ and that the ordering at $z(i)$ of $\mathcal{B}$ is different from that of $z^{\prime}(i)$. We distinguish two cases.

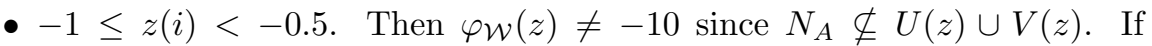
$\varphi_{\mathcal{W}}(z)=-11$ then by $z(i)<z^{\prime}(i)$ and $(\mathrm{M})$ of $\mathcal{W}$ we have $\varphi_{\mathcal{W}}\left(z^{\prime}\right)=-11$ 
and we are done. Suppose $\varphi_{\mathcal{W}}(z) \in\{00,01\}$. Then since at $z(i)$ outcomes $\varphi_{\mathcal{W}}(z)$ and $\varphi_{\mathcal{W}}\left(z^{\prime}\right)$ are indifferent we have $\varphi_{\mathcal{W}}\left(z^{\prime}\right) \in\{00,01\}$. Then, since $N_{B} \subseteq U(z) \cup V(z)$ if and only if $N_{B} \subseteq U\left(z^{\prime}\right) \cup V\left(z^{\prime}\right)$, it follows that $\varphi_{\mathcal{W}}(z)=01$ if and only if $\varphi_{\mathcal{W}}\left(z^{\prime}\right)=01$.

- $z(i)=-0.5$ and $-0.5<z^{\prime}(i) \leq 0$. If $\varphi_{\mathcal{W}}\left(z^{\prime}\right)=-11$, then the indifference between $\varphi_{\mathcal{W}}(z)$ and $\varphi_{\mathcal{W}}\left(z^{\prime}\right)$ at $z^{\prime}(i)$ yields that $\varphi_{\mathcal{W}}(z)=\varphi_{\mathcal{W}}\left(z^{\prime}\right)=-11$. Since $N_{A} \subseteq U\left(z^{\prime}\right) \cup V\left(z^{\prime}\right)$ if and only if $N_{A} \subseteq U(z) \cup V(z)$, it follows that $\varphi_{\mathcal{W}}(z)=-10$ if and only if $\varphi_{\mathcal{W}}\left(z^{\prime}\right)=-10$. Further, since $N_{B} \subseteq$ $U(z) \cup V(z)$ if and only if $N_{B} \subseteq U\left(z^{\prime}\right) \cup V\left(z^{\prime}\right)$, it follows that $\varphi_{\mathcal{W}}(z)=01$ if and only if $\varphi_{\mathcal{W}}\left(z^{\prime}\right)=01$. Hence, we also have $\varphi_{\mathcal{W}}(z)=00$ if and only if $\varphi_{\mathcal{W}}\left(z^{\prime}\right)=00$ since that is the only remaining case. Thus, $\varphi_{\mathcal{W}}(z)=\varphi_{\mathcal{W}}\left(z^{\prime}\right)$.

Finally, we prove FA of $\varphi$. Suppose that all agents weakly prefer $\left(\alpha_{\mathcal{W}}(z), 1\right)$ to $\varphi_{\mathcal{W}}(z)=\left(\alpha_{\mathcal{W}}(z), \beta_{\mathcal{W}}(z)\right)$. It is sufficient to prove that $\beta_{\mathcal{W}}(z)=1$. To the contrary suppose $\beta_{\mathcal{W}}(z)=0$. Then all agents in $N_{B}$ have their dip smaller than or equal to $\frac{1}{2}$. So, $N_{B} \subseteq U(z) \cup V(z)$. As $\beta_{\mathcal{W}}(z)=0$, we have $(U(z), V(z)) \notin \mathcal{W}$. This however contradicts the definition of $\varphi_{\mathcal{W}}$ because if $(U(z), V(z)) \notin \mathcal{W}$ and $N_{B} \subseteq U(z) \cup V(z)$, then $\varphi_{\mathcal{W}}(z)=01$.

We can now formulate the main result of the paper, which is a corollary to the preceding two lemmas.

Corollary 4.5 Let $\varphi$ be a rule. Then $\varphi$ satisfies $S P, C P O, N C$, and FA, if and only if there is a family $\mathcal{W}$ of decisive pairs such that $\varphi=\varphi_{\mathcal{W}}$.

Proof. If $\mathcal{W}$ is a family of decisive pairs, then $\varphi_{\mathcal{W}}$ satisfies SP, CPO, NC, and FA by Lemma 4.4. Conversely, let $\varphi$ satisfy these four conditions. We show that $\varphi=\varphi_{\mathcal{W}_{\varphi}}$, which completes the proof by Lemma 4.3. Let $z \in \mathcal{R}$. Then $\varphi(z)=-11 \Leftrightarrow(U(z), V(z)) \in \mathcal{W}_{\varphi} \Leftrightarrow \varphi_{\mathcal{W}_{\varphi}}(z)=-11$. If $\varphi_{\mathcal{W}_{\varphi}}(z)=01$ then (by the previous step) $\varphi(z) \neq-11$ and moreover, $N_{B} \subseteq U(z) \cup V(z)$, so that $\varphi(z)=01$ by FA. Similarly, $\varphi_{\mathcal{W}_{\varphi}}(z)=-10$ implies $\varphi(z)=-10$. Hence, we also have $\varphi_{\mathcal{W}_{\varphi}}(z)=00$ if and only if $\varphi(z)=00$.

\section{Discussion}

Corollary 4.5 determines the class of all SP, CPO, NC, and FA rules for locating two bads in two neighboring countries. The examples below show that this class leaves room for a spectrum of monotone voting rules between combinations of boundary points, ranging from majority decisions to joint dictatorship. Although not exemplified here, combinations of those over the different countries are possible as well. It shows that the restrictive nature of strategy-proof rules found in the one country case (e.g., Peremans and Storcken, 1999) generalizes to the case of two countries.

Example 5.1 (Majority decisions) Let the pair $(U, V)$ be decisive if and only if $|U| \geq|N \backslash(U \cup V)|$. Thus, a pair is decisive if there are at least as many 
agents who strictly prefer -11 over the other outcomes than there are agents strictly preferring 00,01 or -10 over -11 . Since the agents in $V$ are indifferent between all outcomes we have a (weak) majority for -11 over all other outcomes: -11 is a (weak) Condorcet winner. If $|U|<|N \backslash(U \cup V)|$, then there is a (weak) majority of 00 over all other outcomes, except when $N_{A} \subseteq U \cup V$ or $N_{B} \subseteq U \cup V$. In these cases we have, respectively, that -10 and 00 or 01 and 00 are indifferent to all agents, and FA then imposes the outcome to be -10 or 01 , respectively.

The following example shows that also rules that are highly asymmetrical in agents' decision power, are possible.

Example 5.2 (An almost dictatorial rule) Fix agents $i_{A} \in N_{A}$ and $i_{B} \in$ $N_{B}$. Let the pair $(U, V)$ be decisive both $i_{A}$ and $i_{B}$ are in $U$ or if $N=U \cup V$. If $(U, V)$ is not decisive the outcome is $-10,01$, or 00 , depending on whether, respectively, $N_{A} \subseteq U \cup V, N_{B} \subseteq U \cup V$, or neither of these. In this rule, $i_{A}$ and $i_{B}$ exercise a kind of joint dictatorship. Observe that we cannot have dictatorial rules since these would violate CPO.

The class of rules characterized in Corollary 4.5 does not include rules that allow for independent decisions in the two countries, as we illustrate in the next example.

Example 5.3 (No independent decisions) For a profile $z$ denote $U_{A}(z)=$ $U(z) \cap N_{A}, V_{A}(z)=V(z) \cap N_{A}, U_{B}(z)=U(z) \cap N_{B}$, and $V_{B}=V(z) \cap N_{B}$. At profile $z$ define $\alpha(z)=-1$ if there is a (weak) majority in $N_{A}$ for -1 against 0, i.e., $\left|U_{A}(z)\right| \geq\left|N_{A} \backslash\left(U_{A}(z) \cup V_{A}(z)\right)\right|$ and in all other cases let $\alpha(z)=0$. Define $\beta(z)=1$ if $\left|U_{B}(z)\right| \geq\left|N_{B} \backslash\left(U_{B}(z) \cup V_{B}(z)\right)\right|$. Thus, $\varphi(z)$ is based on (weak) majorities, but now for each country separately. We show that this rule is corruptible. Consider a profile $w$ at which there is a strict majority for 0 in country $B$, that is, $\left|U_{B}(w)\right|<\left|N_{B} \backslash\left(U_{B}(w) \cup V_{B}(w)\right)\right|$; and a strict majority for 0 in country $A$, but with a swing voter at $-\frac{1}{2}$. Then $\varphi(w)=00$. If the swing voter deviates, yielding profile $v$ such that $\left|U_{A}(v)\right|=\left|N_{A} \backslash\left(U_{A}(v) \cup V_{A}(v)\right)\right|$, then $\varphi(v)=-10$. But this swing voter is indifferent between 00 and -10 both at $-\frac{1}{2}$ and at his new dip.

Both FA and NC are needed to deduce that the outcomes of a rule are combinations of country border points. Dropping these conditions may lead to interior solutions - see below. For an analysis of the one agent per country case see Öztürk (2013).

Finally, we provide examples showing the logical independence of the characterizing conditions in Corollary 4.5.

\section{Example 5.4 (Logical independence of SP, CPO, NC, FA)}

Strategy-Proofness Consider a family of decisive pairs $\mathcal{W}$ with associated rule $\varphi_{\mathcal{W}}$. Let $i_{A}, j_{A}$ be two different agents in $N_{A}$ and let $i_{B}, j_{B}$ be two different agents in $N_{B}$. Define the rule $\widehat{\varphi}$ for all profiles equal to $\varphi_{\mathcal{W}}$ except for profiles $q$ at 
which $q\left(i_{A}\right)=-1, q\left(i_{B}\right)=1$, and $q\left(j_{A}\right)=q\left(j_{B}\right)=0$ : then let $\widehat{\varphi}(q)=\left(-\frac{1}{2}, \frac{1}{2}\right)$.

Rule $\widehat{\varphi}$ satisfies FA and CPO, as is not difficult to see. Non-corruptibility can be seen by considering the preferences of the agents $i_{A}, j_{A}, i_{B}, j_{B}$ at such a profile $q$, as given in the following table.

$$
\begin{aligned}
i_{A}: & 00 \sim 01 \succ-\frac{1}{2} \frac{1}{2} \succ-11 \sim-10 \\
j_{A}, j_{B}: & -11 \succ-\frac{1}{2} \frac{1}{2} \succ 00 \sim-10 \sim 01 \\
i_{B}: & 00 \sim-10 \succ-\frac{1}{2} \frac{1}{2} \succ-11 \sim 01
\end{aligned}
$$

Thus, these four agents are not indifferent between the outcome at $q$ and any of the outcomes $-11,-10,00$, or 01 of $\varphi_{\mathcal{W}}$ at a profile not having the structure of $q$. It is not difficult to see that $\widehat{\varphi}$ is not strategy-proof.

Country-Specific Pareto Optimality The constant rule that assigns -11 to every profile satisfies SP, NC, and FA, but not CPO.

Non-Corruptibility The rule in Example 5.3 satisfies SP, CPO, and FA, but not $\mathrm{NC}$.

Far Away Condition Consider a family of decisive pairs $\mathcal{W}$ with associated rule $\varphi_{\mathcal{W}}$. Let rule $\widetilde{\varphi}$ be equal to $\varphi_{\mathcal{W}}$ except that $\widetilde{\varphi}\left(\left(-\frac{1}{2}\right)^{N_{A}},\left(\frac{1}{2}\right)^{N_{B}}\right)=-10$. Then $\widetilde{\varphi}$ violates the far away condition at profile $\left(\left(-\frac{1}{2}\right)^{N_{A}},\left(\frac{1}{2}\right)^{N_{B}}\right)$ for country $B$. Clearly, $\widetilde{\varphi}$ satisfies CPO. For SP and NC consider a unilateral deviation $z$ from $\left(\left(-\frac{1}{2}\right)^{N_{A}},\left(\frac{1}{2}\right)^{N_{B}}\right)$ by agent $i$. First suppose $z(i)<-\frac{1}{2}$. Then CPO implies that, with $\varphi_{\mathcal{W}}(z)=(\alpha(z), \beta(z)), \alpha(z)=0$, so that $(U(z), V(z)) \notin \mathcal{W}$. Since $N_{B} \subseteq U(z) \cup V(z)$, it follows that $\widetilde{\varphi}(z)=01$. At $\left(\left(-\frac{1}{2}\right)^{N_{A}},\left(\frac{1}{2}\right)^{N_{B}}\right)$ agent $i$ is indifferent between -10 and 01 , but at $z(i)$ agent $i$ strictly prefers 01 to -10 . It follows that at these deviations the requirements of SP and $\mathrm{NC}$ hold.

Next consider the case that $0 \geq z(i)>-\frac{1}{2}$. Then $\left(N_{A} \cup N_{B}\right) \subseteq(U(z) \cup V(z))$ and $(U(z), V(z)) \in \mathcal{W}$, which implies that $\widetilde{\varphi}(z)=-11$. At $\left(\left(-\frac{1}{2}\right)^{N_{A}},\left(\frac{1}{2}\right)^{N_{B}}\right)$ agent $i$ is indifferent between -10 and -11 and at $z(i)$ agent $i$ strictly prefers outcome -11 to -10 . Hence, also at these deviations the requirements of SP and NC hold.

Now consider the case that $0 \leq z(i)<\frac{1}{2}$. Then $\left(N_{A} \cup N_{B}\right) \subseteq(U(z) \cup V(z))$ and $(U(z), V(z)) \in \mathcal{W}$, which implies that $\widetilde{\varphi}(z)=-11$. At $\left(\left(-\frac{1}{2}\right)^{N_{A}},\left(\frac{1}{2}\right)^{N_{B}}\right)$ agent $i$ is indifferent between -10 and -11 and at $z(i)$ agent $i$ strictly prefers -11 to -10 . Hence, at these deviations again the requirements of SP and NC hold.

Finally, consider $z(i)>\frac{1}{2}$. CPO implies $\beta(z)=0$. Hence, $(U(z), V(z)) \notin \mathcal{W}$. Since $N_{A} \subseteq U(z) \cup V(z)$, it follows that $\widetilde{\varphi}(z)=-10$. Again the requirements of SP and NC hold at these deviations.

\section{References}

Barbera S, Berga D, Moreno B (2012) Domains, ranges and strategy-proofness: the case of single-dipped preferences. Social Choice and Welfare 39:335352 
Gibbard A (1973) Manipulation of voting schemes: A general result. Econometrica 41:587-602

Lescop D (2007) Optimal mechanisms for siting noxious facilities. Review of Economic Design 10:273-284

Manjunath V (2014) Efficient and strategy-proof social choice when preferences are single-dipped. International Journal of Game Theory 43:579-597

Öztürk M (2013) Strategy-proofness and single-dipped preferences. PhD Thesis, Maastricht University

Öztürk M, Peters H, Storcken T (2013) Strategy-proof location of a public bad on a disc. Economics Letters 119:14-16

Öztürk M, Peters H, Storcken T (2014) On the location of public bads: strategyproofness under two-dimensional single-dipped preferences. Economic Theory 56:83-108

Peremans W, Storcken T (1999) Strategy-proofness on single-dipped preference domains. In de Swart HMC (ed.) Logic, Game theory and Social choice. Tilburg University Press, The Netherlands

Ritz S (1985) Restricted domains, Arrow social choice functions and noncorruptible and nonmanipulable social choice correspondences: the case of private and public alternatives. Journal of Economic Theory 35:1-18

Sakai T (2012) Fair waste pricing: an axiomatic analysis to the NIMBY problem. Economic Theory 50:499-521

Satterthwaite MA (1975) Strategy-proofness and Arrow's conditions: Existence and correspondence theorems for voting procedures and social welfare functions. Journal of Economic Theory 10:187-207 